\title{
Social cohesion and integration in schools reduces suicidal behaviour rate
}

\author{
By Jessica K. Edwards
}

Emerging data suggest that strengthening positive social bonds and improving social integration might reduce suicidal behaviours in youthı to date; little research has studied the effect of social integration, on suicide behaviours, with reference to a young person's social network structure - namely, an individual's position within their network and the patterns of relationships among members of the network. Researchers in the USA have now addressed this knowledge gap by examining whether structural characteristics of school networks (including both peer and adult connections) can predict school rates of suicide ideation (SI) and/or suicide attempts (SA). The study included $>10,000$ students from 38 US high schools who answered questions about suicidal thoughts and behaviours (STB). The data showed that schools with friendship networks reflecting greater integration and cohesion had lower rates of SI and SA. Specifically, students with more friendship ties, who were part of larger, interconnected friendship groups, were less likely to report SI and SA. These indices aggregated at the school population level and predicted lower school rates of suicidal thoughts and behaviours. The researchers also found evidence of a dose-response relationship, suggesting that the impact of low integration and cohesion occurs along a continuum, increasing vulnerability for SI and, at higher levels, for SA. Finally, they found that student isolation from adults, if youth-adult relationships were concentrated in fewer students, and higher popularity of suicidal youth, were associated with higher SA rates. Going forward, the researchers believe that network-informed suicide prevention approaches could be developed and tested in schools and other education settings and that protective peer and youth-adult bonds, group cohesion, and the social influence of healthy, coping youth could be maximised.

Wyman, P. et al. (2019), Peer-adult network structure and suicide attempts in 38 high schools: implications for network informed suicide prevention. J. Child Psychol. Psychiatr. doi:10.1111/jcpp.13102

\section{References}

${ }^{1}$ King, C. A. and Merchant, C. R. (2008). Social and interpersonal factors relating to adolescent suicidality:

a review of the literature. Arch. Suicide Res. 12, 181-196. doi: 10.1080/13811110802101203 